\title{
New oral anticoagulants-what the cardiothoracic surgeon needs to know
}

\author{
Tsuyoshi Kaneko, MD, Maroun Yammine, MD, and Sary F. Aranki, MD
}

See related commentary on pages $1802-3$.

Supplemental material is available online.

Oral anticoagulation for cardiothoracic patients has traditionally been synonymous with the use of warfarin. The recent introduction of new oral anticoagulants (NOACs) targeting factor $\mathrm{Xa}$ or thrombin represents a new approach for anticoagulation. Cardiothoracic surgeons need to familiarize themselves with these agents, because more preoperative patients will be taking NOACs. Thus, strategies for discontinuation before surgery with or without bridging have become paramount. The rapid onset of action, wide therapeutic index, and a steady therapeutic state without the need for monitoring has made these new agents more attractive than warfarin for these indications.

The currently available NOACs are dabigatran etexilate (Pradaxa; Boehringer Ingelheim, Ridgefield, Conn), rivaroxaban (Xarelto; Bayer HealthCare AG, Leverkusen, Germany), apixaban (Eliquis; Bristol-Myers Squibb, New York, NY), and edoxaban (Savaysa; Daiichi Sankyo, Tokyo, Japan; not approved in the United States). We have reviewed the pharmacologic profile, clinical evidence for safety and efficacy, currently approved indications, and strategies to guide in the perioperative treatment of patients taking NOACs.

\section{TRADITIONAL ORAL ANTICOAGULANT- WARFARIN}

Warfarin was approved for human use in 1954 and has been the mainstay oral anticoagulant in clinical practice ever since, until recently. Warfarin inhibits vitamin K-dependant synthesis of calcium-dependant clotting factors and the regulatory proteins $\mathrm{C}$ and $\mathrm{S}$. Multiple food-drug and drug-drug

From the Division of Cardiac Surgery, Brigham and Women's Hospital, Boston, Mass.

Disclosures: Authors have nothing to disclose with regard to commercial support.

Received for publication March 24, 2014; revisions received May 18, 2014; accepted

for publication May 22, 2014; available ahead of print June 25, 2014.

Address for reprints: Sary F. Aranki, MD, Division of Cardiac Surgery, Brigham and

Women's Hospital, 75 Francis St, Boston, MA (E-mail: saranki@partners.org).

J Thorac Cardiovasc Surg 2014;148:1794-801

0022-5223/\$36.00

Copyright (c) 2014 by The American Association for Thoracic Surgery

http://dx.doi.org/10.1016/j.jtcvs.2014.05.060 interactions with food and other medications has complicated the use of warfarin (Table 1 and Appendix 1). The narrow therapeutic index with frequent monitoring has been a burden of therapy because of the frequent dose adjustment needed, and the long half-life of warfarin (approximately 60 hours) requires several days to restore the therapeutic anticoagulation level after interruption.

One major advantage compared with NOACs is its reversibility. In an emergency setting, warfarin can be reversed with fresh frozen plasma, and patients can undergo a procedure safely. Vitamin K provides excess cofactor for ongoing coagulation factor carboxylation in the setting of irreversible inhibition by warfarin and, therefore, requires synthesis of new coagulation factors to have an effect. For additional information on warfarin, see Appendix 1.

These limitations have led to development of NOACs targeting factor Xa or thrombin (factor IIa).

\section{USE OF WARFARIN IN CARDIOTHORACIC SURGERY}

We reviewed the use of warfarin after cardiac surgery at Brigham and Women's Hospital. In 2011, of 1257 cardiac cases performed, 586 patients $(46.6 \%)$ were discharged with warfarin. Similarly, in 2012, of 1234 cardiac cases performed, 557 patients $(45.1 \%)$ were discharged with warfarin. These data included all patients who were taking warfarin; hence, both patients who were taking it preoperatively and had started taking it postoperatively were counted.

\section{PHARMACOKINETIC AND PHARMACODYNAMIC PROFILE OF ORAL ANTICOAGULANTS}

The characteristics of dabigatran, rivaroxaban, apixaban, and edoxaban are compared with warfarin in Table 1.

\section{Dabigatran}

Dabigatran etexilate is a direct thrombin inhibitor and comes in the form of a prodrug with a bioavailability of $7.2 \%{ }^{1}$ It is converted to the active form dabigatran in the liver. It directly blocks the active site and prevents conversion of fibrinogen into fibrin. The half-life of dabigatran is about 13 hours in healthy individuals and is cleared by the kidneys (renal excretion, 80\%). Renal function should be monitored in patients with renal insufficiency, because poor renal function can impair renal excretion and prolong the effect of anticoagulation. Accordingly, the dose adjustment should be determined by the creatinine clearance (Table 1). Interaction with 


\section{Abbreviations and Acronyms \\ $\mathrm{AF} \quad=$ atrial fibrillation \\ ARISTOTLE $=$ Apixaban for Reduction in Stroke and Other Thromboembolic \\ Events in Atrial Fibrillation \\ ENGAGE $=$ Effective Anticoagulation with \\ AF-TIMI 48 Factor Xa Next Generation in \\ Atrial Fibrillation-Thrombolysis \\ in Myocardial Infarction 48 \\ FDA $\quad=$ Food and Drug Administration \\ INR $=$ international normalized ratio \\ NOAC $=$ new oral anticoagulant \\ $\mathrm{p}$-gp $\quad=$ permeability glycoprotein \\ RE-ALIGN = randomized, phase II study to \\ evaluate the safety and \\ pharmacokinetics of oral \\ dabigatran etexilate in patients after heart valve replacement \\ RE-LY = Randomized Evaluation of Long- \\ Term Anticoagulation Therapy \\ ROCKET AF = Rivaroxaban Once-daily Oral \\ Direct Factor Xa Inhibition \\ Compared with Vitamin K \\ Antagonism for Prevention of \\ Stroke and Embolism Trial in \\ Atrial Fibrillation}

permeability glycoprotein (p-gp) inhibitors such as quinidine, ketoconazole, and verapamil can increase the plasma concentration by reducing the clearance of dabigatran etexilate. ${ }^{2}$ This is more significant in patients with renal failure, and patients with severe renal impairment (creatinine clearance $<30 \mathrm{~mL} / \mathrm{min}$ ) should not be taking a p-gp inhibitor and dabigatran. Because the plasma concentration and anticoagulant effect are dose dependent and predictable, it does not require repeated monitoring, unlike warfarin. The peak effect occurs within 0.5 to 2 hours, with a steady state achieved within 3 days. ${ }^{3}$ It is known that a plasma trough dabigatran level of $<50 \mathrm{ng} / \mathrm{mL}$ is associated with an increased risk of thromboembolism ${ }^{4}$; however, the trough level is not commonly measured clinically at this point.

\section{Rivaroxaban}

Rivaroxaban, which is derived from 2-oxazolidone, is an oral inhibitor of factor $\mathrm{Xa}$, binding reversibly to its active site. Rivaroxaban has an oral bioavailability of $80 \%$, and a half-life of 7 to 11 hours; $67 \%$ is cleared renally but approximately one half of that is excreted unchanged in the urine. ${ }^{5}$ In addition to interaction with $\mathrm{p}$-gp inhibitors, rivaroxaban is metabolized by way of CYP3A4; thus, strong inhibitors of CYP3A4, such as human immunodeficiency virus protease inhibitors, macrolide antibiotics (erythromycin), and azole antifungal agents (eg, ketoconazole), should be avoided because these agents will increase the risk of bleeding. No monitoring is required for rivaroxaban. It is the only NOAC approved by the Food and Drug Administration (FDA) approved for once daily dose.

\section{Apixaban}

Apixaban is an oral direct inhibitor of factor $\mathrm{Xa}$, and its bioavailability is $\leq 66 \%$. Its half-life is approximately 12.7 hours, and $25 \%$ of the drug is cleared renally. ${ }^{6}$ Administration of a strong dual inhibitor of CYP3A4 plus a p-gp (ie, ketoconazole, itraconazole, ritonavir, or clarithromycin) will increase the risk of bleeding and should be avoided.

\section{Edoxaban}

Edoxaban is also an oral direct inhibitor of factor Xa. Its bioavailability is $62 \%$, and the half-life is 10 to 14 hours, which allows daily dosing. Approximately $50 \%$ of drug is renally excreted. Because Edoxaban is p-gp transporter, it has interactions with strong p-gp inhibitors but minimal interaction with CYP3A4 inhibitors. ${ }^{7}$

\section{Interactions With Amiodarone and Dronedarone}

It is worth emphasizing that because amiodarone is a moderate $\mathrm{p}$-gp inhibitor and dronedarone a strong $\mathrm{p}$-gp inhibitor, using dabigatran, rivaroxaban, and apixaban for patients with either amiodarone or dronedarone can increase the effect of anticoagulation about $50 \%$ and, therefore, should be avoided. ${ }^{2}$ This effect is smaller with edoxaban, and amiodarone and edoxaban can be administered if one of the doses is decreased. In contrast, dronedarone is a strong p-gp inhibitor, and the dose of edoxaban should be reduced by $50 \%$ if dronedarone is co-administered. ${ }^{7}$ Safe concomitant use of amiodarone and apixaban have been reported.

The currently approved indications and dosage for NOACs are listed in Table 1 and Appendix 1. Advantages and disadvantages of NOACs compared with warfarin are listed in Table 2.

\section{CLINICAL EVIDENCE FOR NOACS Prevention of Stroke in Patients With Atrial Fibrillation}

Dabigatran, rivaroxaban, and apixaban have been approved by the FDA for prevention of stroke in patients with nonvalvular atrial fibrillation $(\mathrm{AF})$ on the basis of the following results.

The Randomized Evaluation of Long-Term Anticoagulation Therapy (RE-LY) trial compared 2 doses of dabigatran (110 mg twice daily or $150 \mathrm{mg}$ twice daily) with warfarin (international normalized ratio [INR], 2-3) for stroke prevention in patients with nonvalvular $\mathrm{AF}$ and $\geq 1$ risk factor for stroke. ${ }^{9}$ For the prevention of the composite outcome of 
TABLE 1. Characteristics of new anticoagulants compared with warfarin

\begin{tabular}{|c|c|c|c|c|c|}
\hline Variable & Warfarin & Dabigatran & Rivaroxaban & Apixaban & Edoxaban \\
\hline $\begin{array}{l}\text { Mechanism of } \\
\text { action }\end{array}$ & Vitamin $\mathrm{K}$ antagonist & Thrombin inhibitor & Factor Xa inhibitor & Factor Xa inhibitor & Factor $\mathrm{Xa}$ inhibitor \\
\hline $\mathrm{T} 1 / 2(\mathrm{~h})$ & $\leq 40$ & 13 & $7-11$ & 12.7 & $10-14$ \\
\hline Excretion & $\begin{array}{l}\text { Renal (metabolized in } \\
\text { liver) }\end{array}$ & Renal $(80 \%)$ & $\begin{array}{l}\text { Renal }(66 \%) / \\
\text { hepatobiliary }\end{array}$ & Renal $(25 \%) /$ fecal & Renal $(50 \%) /$ fecal \\
\hline Bioavailability (\%) & $\leq 95$ & 7.2 & $\leq 80$ & $\leq 66$ & 62 \\
\hline Drug interactions & Multiple (see Appendix 1) & Inhibitors of $\mathrm{p}$-gp & $\begin{array}{c}\text { Inhibitors of p-gp } \\
\text { and CYP3A4 }\end{array}$ & $\begin{array}{c}\text { Inhibitors of p-gp } \\
\text { and CYP3A4 }\end{array}$ & Inhibitors of $\mathrm{p}-\mathrm{gp}$ \\
\hline Food interactions & $\begin{array}{l}\text { Leafy green vegetables } \\
\text { (high in vitamin K), } \\
\text { alcohol, cranberry juice }\end{array}$ & No & No & No & No \\
\hline Monitoring & Yes & No & No & No & No \\
\hline Approved dose & Variable & $\begin{array}{l}150 \mathrm{mg} \text { bid (AF); } \\
110 \mathrm{mg} \text { bid } \\
\text { (AF-Europe) }\end{array}$ & $\begin{array}{l}10 \mathrm{mg} \text { daily (VTE) } \\
20 \mathrm{mg} \text { daily (AF) } \\
15 \mathrm{mg} \text { bid for first } 21 \mathrm{~d} \text {, } \\
\text { then } 20 \mathrm{mg} \text { daily } \\
\text { (treatment of DVT) }\end{array}$ & $5 \mathrm{mg}$ bid (AF) & $\begin{array}{l}30 \text { mg daily (VTE, } \\
\text { Japan) } 60 \mathrm{mg} \text { daily } \\
\text { for VTE, } 30 \text { or } 60 \mathrm{mg} \\
\text { daily for AF (FDA } \\
\text { and Europe pending } \\
\text { approval) }\end{array}$ \\
\hline Dose reduction & Depending on INR & $\begin{array}{l}75 \mathrm{mg} \text { bid for } \mathrm{CrCl} \\
30-50 \mathrm{~mL} / \mathrm{min} \\
\text { Consider stopping } \\
\text { for acute renal } \\
\text { failure (AF) } \\
\text { Avoid use with } \\
\text { p-gp inhibitor, } \\
\text { rifampin }\end{array}$ & $\begin{array}{l}15 \mathrm{mg} \text { daily for } \mathrm{CrCl} \\
15-50 \mathrm{~mL} / \mathrm{min}(\mathrm{AF}) \\
\text { Avoid use in strong } \\
\text { dual inhibitor of } \\
\text { p-gp and CYP3A4 }\end{array}$ & $\begin{array}{l}2.5 \mathrm{mg} \text { bid (AF) for } \geq 2 \\
\text { of the following: age } \\
>80 \mathrm{y}, \text { body weight } \\
<60 \mathrm{~kg}, \mathrm{Cr}>1.5 \mathrm{mg} / \mathrm{dL} \\
2.5 \mathrm{mg} \text { bid or stop when } \\
\text { used with strong dual } \\
\text { inhibitor of p-gp and } \\
\text { CYP3A4 }\end{array}$ & $\begin{array}{l}\text { Reduce to half dose for } \\
\mathrm{CrCl} 30-50 \mathrm{~mL} / \mathrm{min} \text {, } \\
\text { weight } \leq 60 \mathrm{~kg} \text {, or } \\
\text { were taking a potent } \\
\text { p-gp inhibitor }\end{array}$ \\
\hline $\begin{array}{l}\text { Approved } \\
\text { indications (FDA) }\end{array}$ & $\begin{array}{l}\text { Prevention and treatment } \\
\text { of VTE, AF, } \\
\text { mechanical valves }\end{array}$ & $\begin{array}{l}\text { Nonvalvular AF, } \\
\text { treatment of DVT, } \\
\text { prevention of VTE } \\
\text { recurrence }\end{array}$ & $\begin{array}{l}\text { VTE prevention after hip } \\
\text { or knee replacement, } \\
\text { nonvalvular AF, } \\
\text { treatment of DVT, } \\
\text { prevention of VTE } \\
\text { recurrence }\end{array}$ & $\begin{array}{l}\text { Nonvalvular AF, VTE } \\
\text { prevention after hip } \\
\text { or knee replacement }\end{array}$ & Not approved \\
\hline
\end{tabular}

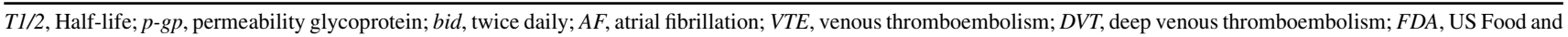
Drug Administration; INR, international normalized ratio; $\mathrm{CrCl}$, creatinine clearance; $\mathrm{Cr}$, creatinine.

stroke or systemic embolism, dabigatran $110 \mathrm{mg}$ twice daily was not inferior to warfarin, and dabigatran $150 \mathrm{mg}$ twice daily was superior to warfarin. The incidence of intracranial, life-threatening, or any type of bleeding was reduced in both dabigatran dosage groups, and low-dose dabigatran also reduced the incidence of major bleeding. The high-dose dabigatran group had a greater incidence of gastrointestinal bleeding. The original report had a significantly greater incidence statistically of myocardial infarction in the 150-mg dabigatran group compared with the warfarin group; however, after readjudication of myocardial infarction, the difference was nonsignificant. ${ }^{10}$ The FDA did not approve the 110-mg twice daily dose, however, citing that they could not identify a subgroup of patients for whom the lower dose would be preferred over the higher dose. ${ }^{11}$

The Rivaroxaban Once-daily Oral Direct Factor Xa Inhibition Compared with Vitamin K Antagonism for Prevention of Stroke and Embolism Trial in Atrial
Fibrillation (ROCKET AF) compared rivaroxaban $20 \mathrm{mg}$ daily with warfarin (INR, 2-3). ${ }^{12}$ For the primary endpoint of stroke and systemic embolism, rivaroxaban was not inferior to warfarin. The rates of major and nonmajor clinically relevant bleeding events were similar, and significant reductions in the rate of intracranial hemorrhage and fatal bleeding occurred in the rivaroxaban group. However, an increase in major gastrointestinal bleeding was observed in the rivaroxaban group.

The Apixaban for Reduction in Stroke and Other Thromboembolic Events in Atrial Fibrillation (ARISTOTLE) trial compared apixaban $5 \mathrm{mg}$ twice daily with warfarin (INR, 2-3). ${ }^{13}$ Apixaban was superior to warfarin for the primary endpoint of the composite outcome of stroke and systemic embolism. The rate of major bleeding events, intracranial hemorrhage, and all-cause mortality were all significantly lower in the apixaban group. No difference was seen in the incidence of gastrointestinal bleeding between the 2 groups. 
The Effective Anticoagulation with Factor Xa Next Generation in Atrial Fibrillation-Thrombolysis in Myocardial Infarction 48 (ENGAGE AF-TIMI 48) trial was recently published. That trial compared $30 \mathrm{mg}$ and $60 \mathrm{mg}$ once daily edoxaban to warfarin. ${ }^{14}$ Edoxaban in both dosages was not inferior to warfarin when compared using the primary endpoint of stroke or systemic embolism. The annualized rates of major bleeding and causes of cardiovascular death were significantly lower in both edoxaban groups compared with warfarin. Major gastrointestinal bleeding was less frequent with low-dose edoxaban than with warfarin but was more frequent with high-dose edoxaban than with warfarin.

A recent meta-analysis of all 4 randomized warfarincontrolled studies showed that NOACs significantly reduced stroke or systemic embolic events by $19 \%$ compared with warfarin, mainly by reducing hemorrhagic stroke. ${ }^{15}$ NOACs also reduced all-cause mortality and intracranial hemorrhage but resulted in an increased incidence of gastrointestinal bleeding. See Appendix 1 for the incidence of gastrointestinal bleeding with the use of NOACs. Adverse events of NOACs in major atrial fibrillation trial are summarized in Table 3.

\section{Anticoagulation for Mechanical Heart Valves}

An interest has developed to use NOACs for anticoagulation in those with prosthetic heart valves. Dabigatran is the only drug that has been studied in this patient population to date.

A large phase II randomized controlled study, namely the randomized, phase II study to evaluate the safety and pharmacokinetics of oral dabigatran etexilate in patients after heart valve replacement (RE-ALIGN) trial, was initiated in 2012. ${ }^{16}$ That trial included patients who had undergone implantation of a mechanical valve (aortic or mitral) or implantation of a mitral bileaflet valve $<3$ months before randomization. The trial was terminated prematurely owing to an increase in stroke ( $5 \%$ vs $0 \%$ ), myocardial infarction, and major bleeding $(4 \%$ vs $2 \%)$ in the dabigatran group compared with the warfarin group. Thus, the FDA announced in December 2012 that dabigatran is contraindicated for patients with mechanical heart valves.

Several criticisms pertain to that trial. One is that nearly $80 \%$ of patients in that trial had undergone recent surgery before the initiation of dabigatran. Early thrombogenicity after surgery is well known, and this could have affected the anticoagulation level. Another is the trough target of $50 \mathrm{ng}$, which was calculated from RE-LY trial. The mean age for RE-LY trial was 71 years compared with 56 years in the RE-ALIGN study and caused a lower than projected plasma level of dabigatran in the first 4 weeks. A future trial with a higher trough target and an initial period of dose adjustment might be needed to assess the true validity.

Currently, dabigatran is contraindicated and should not be used for patients with mechanical valves. For bioprosthetic valves, the FDA made the following statement: "The use of dabigatran in patients with bioprosthetic valves has not been evaluated and cannot be recommended." No large study of rivaroxaban, apixaban, and edoxaban for patients with a prosthetic valve has been performed, and their use in heart prosthesis is not recommended. For other FDA-approved indications, see Appendix 1 for the clinical evidence.

\section{NONVALVULAR AF}

NOACs are only approved for patients with AF that is "nonvalvular" in origin; however, nonvalvular AF has not been consistently defined among the trials. Thus, questions remain, including whether moderate mitral regurgitation with $\mathrm{AF}$ denotes nonvalvular $\mathrm{AF}$; and whether $\mathrm{AF}$ in the presence of a bioprosthetic valve should be considered nonvalvular AF. In the randomized controlled trials that assessed the validity of NOACs for stroke prevention in patients with $\mathrm{AF}$, the exclusion criteria were as follows: hemodynamically relevant valvular disease and a prosthetic valve in the RE-LY trial, ${ }^{9}$ hemodynamically significant mitral stenosis and a prosthetic valve in the ROCKET-AF trial, ${ }^{12}$ moderate or severe mitral stenosis and a prosthetic valve in the ARISTOTLE trial, ${ }^{13}$ and moderate to severe mitral stenosis in the ENGAGE AF-TIMI 48 trial. ${ }^{14}$ Thus, the definitions were most restrictive in the RE-LY trial and most permissive in the ENGAGE AF-TIMI 48 trial. It is also important to add that previous mitral valve repair and annular rings were not exclusion criteria in these trials. Recently published American College of Cardiology/ American Heart Association guidelines for the management of $\mathrm{AF}$ have defined nonvalvular $\mathrm{AF}$ as that not associated with a mechanical or bioprosthetic valve or mitral stenosis. ${ }^{17}$

\section{PERIOPERATIVE ISSUES}

\section{Stopping NOACs Before Surgery}

Recommendations on the timing of discontinuation of NOACs before surgery have been published. ${ }^{18,19}$ The timing of discontinuation depends on 3 factors: the half-life of the drug, the patient's renal function, and the type of surgery. Figure 1 shows the suggested management of preoperative discontinuation of NOAC. ${ }^{20}$ Cardiothoracic surgery is considered major surgery; hence, more complete reversal is required preoperatively. The last dose should be given 4 to 5 half-lives of the drug between the last dose and surgery, which corresponds to the minimal amount of residual anticoagulation effect (3\%-6\%). The latter will differ according to the patient's renal function. In patients undergoing major surgery (eg, cardiac, 


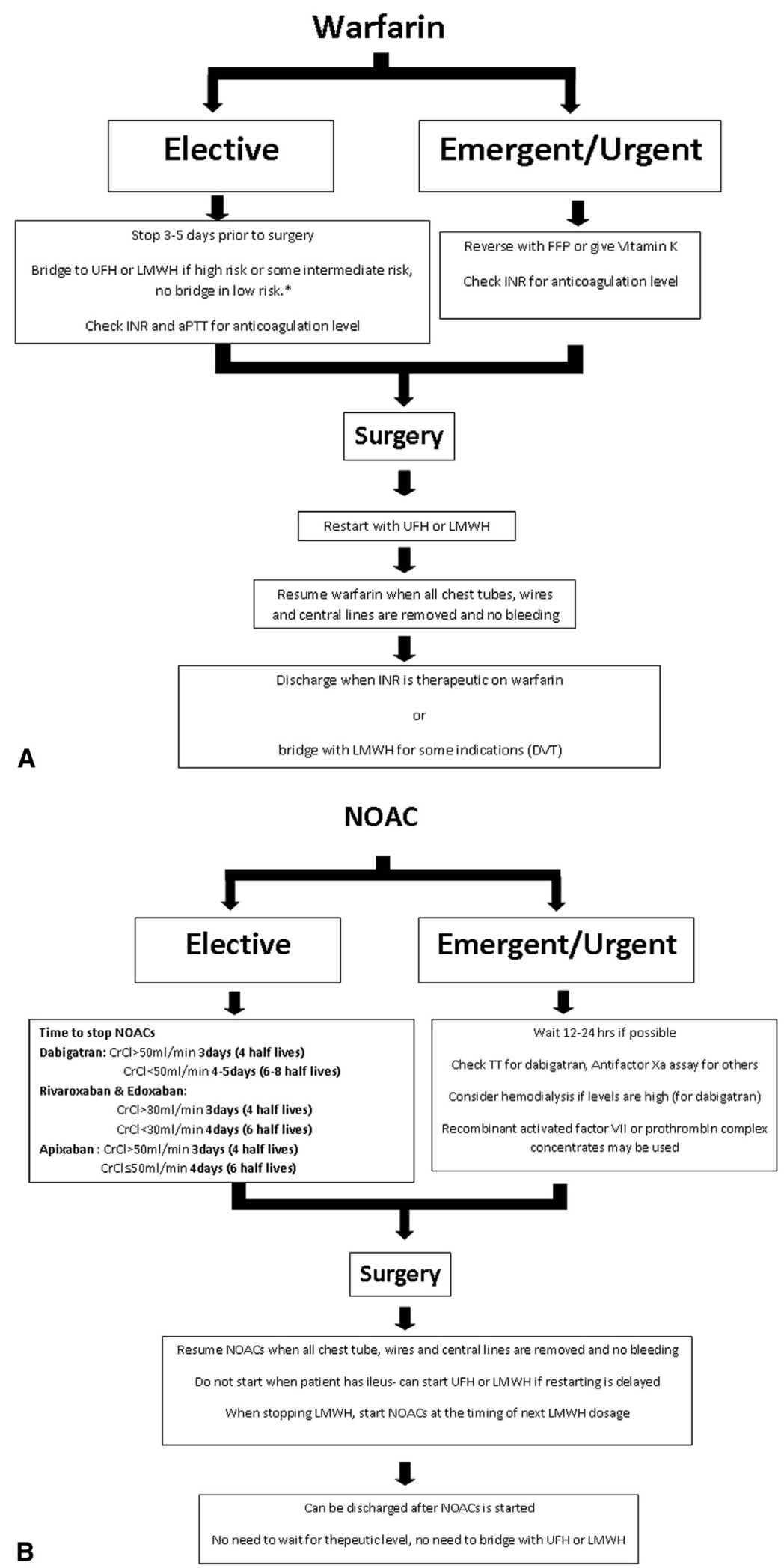

FIGURE 1. Perioperative management of a patient undergoing major cardiothoracic surgery who is taking (A) warfarin or (B) new oral anticoagulants (NOACs). UFH, Unfractionated heparin; $L M W H$, low-molecular-weight heparin; INR, international normalized ratio; aPTT, activated partial thromboplastin time; FFP, fresh frozen plasma; $D V T$, deep venous thrombosis; $\mathrm{CrCl}$, creatinine clearance; $T T$, thrombin time. *Risk stratification according to American College of Chest Physician 2012 guidelines. ${ }^{27}$ 
TABLE 2. Advantages and disadvantages of NOACs compared with warfarin

\begin{tabular}{l}
\hline Advantages \\
Freedom from frequent blood monitoring and dose adjustments \\
Fewer interaction with food and other medications \\
Fast onset and offset \\
Rapidly cleared after discontinuation and easier to manage \\
preoperatively \\
Reducing thrombosis and fatal bleeding \\
Disadvantages \\
Lack of reversal agent \\
Higher cost per pill \\
Underdosing when patient forgets to take medication \\
Dose adjustments required for patients with renal failure \\
Contraindicated for valve prosthesis, pregnancy, children, and patients \\
requiring dialysis \\
Contraindicated for those with neuraxial anesthesia or spinal puncture \\
\hline
\end{tabular}

thoracic, gastrointestinal, neurosurgery) with normal or mildly impaired renal function (creatinine clearance $>$ $50 \mathrm{~mL} / \mathrm{min}$ ), the last dose should be given 3 days before surgery, because 48 to 60 hours of interruption will lead to a minimal anticoagulant effect of $<3 \%$ to $6 \%$ at surgery. The dose adjustments stratified by renal function are highlighted in Figure 1. For minor surgery, such as bronchoscopy or pacemaker insertion, a mild to moderate anticoagulation effect of $<12 \%$ to $25 \%$ has been accepted at surgery and can be achieved by stopping the NOAC 2 days before the procedure in patients with normal renal function.

Although these approaches are reasonable according to the pharmacokinetic profiles, the only supportive empirical evidence has comes from the RE-LY trial, a randomized controlled study between dabigatran and warfarin for stroke prevention in patients with nonvalvular $\mathrm{AF}$, in which a protocol similar to that shown in Figure 1 was adopted for patients undergoing a major procedure. ${ }^{9}$ In that study, 4500 patients had had their anticoagulation interrupted. No statistically significant difference was found in perioperative major bleeding among the dabigatrantreated patients $(3.8 \%$ with the $110-\mathrm{mg}$ dose, $5.1 \%$ with the 150-mg dose) and warfarin-treated patients $(4.6 \%)$.

\section{Monitoring of NOACs}

NOACs do not require routine monitoring; however, in the case of emergency surgery or acute bleeding, blood monitoring tests can be used.

For dabigatran, the diluted thrombin time can be determined using the Hemoclot assay (Boehringer Ingelheim Pharmaceuticals, Ingelheim, Germany). That assay provides direct assessment of thrombin activity and can be performed before surgery. A diluted thrombin time of $<65$ is ideal before surgery to decrease the risk of bleeding. ${ }^{21}$ If the diluted thrombin time is not available, the activated partial thromboplastin time or prothrombin time can be used to monitor dabigatran to achieve a goal target similar to that of other anticoagulants (heparin) but has a lower slope, with an increase in concentration, is less reliable than the thromboplastin time, and must be used with caution. ${ }^{22}$

For factor Xa inhibitors, antifactor Xa assay can be performed for rivaroxaban and apixaban. The prothrombin time can also be used, although it is less reliable. ${ }^{23}$ The factor Xa assay can estimate the level of factor Xa inhibitors; however, no data have shown a safe level for bleeding. The activated partial thromboplastin time has been unpredictable in factor Xa inhibitors. ${ }^{23}$

\section{Reversing the Effect of NOACs}

Currently, no reversal agent is available for a NOAC, although a phase II trial is underway for a dabigatran reversal agent. ${ }^{24}$ In the case of major bleeding, the NOACs must first be stopped. The source of bleeding must be controlled, surgically if needed, and the urine output must be maintained to continue renal excretion. It is important to remember that the use of fresh frozen plasma does not reverse the effect of an NOAC.

If these measures fail, hemodialysis or other renal replacement therapy can be considered in hemodynamically stable patients taking dabigatran owing to its relatively low plasma protein binding $(-35 \%)$ and high rate of renal excretion $(80 \%))^{6}$ How effective dialysis would be in the case of factor Xa inhibitors, each of which has a considerably greater rate of protein binding, is not known. Activated coagulation factors and prothrombin complex are currently recommended in the reversal of the NOAC effect. Small reports have been published of factor VIIa recombinant reversing the activity of dabigatran and rivaroxaban, ${ }^{25}$ and in vivo studies are ongoing to confirm the effect of prothrombin complex concentrate. A reversal agent for factor Xa (Andexanet alfa, PRT4445; Portola Pharmaceutical, South San Francisco, Calif) and a synthetic small molecule reversal agent (PER977; Perosphere, Inc, Danbury, Conn) are in late stage development.

\section{Restarting Anticoagulants Postoperatively}

Resuming NOACs after surgery must be done with caution. The rapid onset of action, with a peak level occurring 1 to 3 hours after ingestion, can cause significant bleeding in cases of incomplete hemostasis. Also, postoperative bowel dysmotility and acid suppression therapy can affect the absorption of the medication. Although direct evidence is lacking, resuming NOACs beyond the second or third day after major cardiothoracic surgery, after complete hemostasis and removal of the chest tube and pacing wires, is recommended. ${ }^{13}$ 
TABLE 3. Adverse events of NOACs in major atrial fibrillation trial (all hazard ratios of stroke and systemic embolism obtained from noninferiority analysis)

\begin{tabular}{|c|c|c|c|c|c|c|c|c|c|c|}
\hline \multirow[b]{3}{*}{ Variable } & \multicolumn{3}{|c|}{ RE-LY trial } & \multicolumn{2}{|c|}{ ARISTOTLE trial } & \multicolumn{3}{|c|}{ ENGAGE trial } & \multicolumn{2}{|c|}{ ROCKET AF trial } \\
\hline & \multirow[b]{2}{*}{ Warfarin } & \multicolumn{2}{|c|}{ Dabigatran $^{9}$ (mg bid) } & \multirow{2}{*}{ Warfarin } & \multirow{2}{*}{$\begin{array}{c}\text { Apixaban }^{12} \\
(5 \mathrm{mg} \text { bid })\end{array}$} & \multirow{2}{*}{ Warfarin } & \multicolumn{2}{|c|}{$\operatorname{Edoxaban}^{13}$ (mg bid) } & \multirow[b]{2}{*}{ Warfarin } & \multirow{2}{*}{$\begin{array}{c}\text { Rivaroxaban }^{14} \\
\text { (20 mg daily) }\end{array}$} \\
\hline & & 110 & 150 & & & & 30 & 60 & & \\
\hline Total patients & 6022 & 6015 & 6076 & 9081 & 9120 & 7036 & 7034 & 7035 & 7133 & 7131 \\
\hline $\begin{array}{l}\text { Stroke and systemic embolism } \\
\quad(\% / y)\end{array}$ & 1.69 & 1.53 & 1.11 & 1.6 & 1.27 & 1.5 & 1.61 & 1.18 & 2.4 & 2.1 \\
\hline $\begin{array}{l}\text { HR for stroke and systemic } \\
\text { embolism vs warfarin }\end{array}$ & & $0.91^{*}$ & 0.66 & & 0.79 & & $1.07 \dagger$ & $0.79 \dagger$ & & $0.88^{*}$ \\
\hline Ischemic stroke $(\% / y)$ & 1.2 & 1.32 & 0.92 & 1.05 & 0.97 & 1.25 & 1.77 & 1.25 & 1.42 & 1.34 \\
\hline $\begin{array}{l}\text { HR for ischemic stroke vs } \\
\text { warfarin }\end{array}$ & & $1.11^{*}$ & 0.76 & & $0.92 *$ & & 1.41 & $1.00^{*}$ & & $0.94 *$ \\
\hline Hemorrhagic stroke $(\% / y)$ & 0.38 & 0.12 & 0.10 & 0.47 & 0.24 & 0.47 & 0.16 & 0.26 & 0.44 & 0.26 \\
\hline $\begin{array}{l}\text { HR for hemorrhagic } \\
\text { stroke vs warfarin }\end{array}$ & & 0.31 & 0.26 & & 0.51 & & 0.33 & 0.54 & & 0.59 \\
\hline Major bleeding (\%/y) & 3.36 & 2.71 & 3.11 & 1.69 & 0.96 & 3.43 & 1.61 & 2.75 & 5.42 & 5.55 \\
\hline $\begin{array}{l}\text { HR for major bleeding } \\
\text { vs warfarin }\end{array}$ & & 0.80 & $0.93 *$ & & 0.57 & & 0.47 & 0.80 & & $1.04 *$ \\
\hline Intracranial bleeding $(\% / y)$ & 0.74 & 0.23 & 0.30 & 0.80 & 0.33 & 0.85 & 0.26 & 0.39 & 1.18 & 0.77 \\
\hline $\begin{array}{l}\text { HR for intracranial } \\
\text { bleeding vs warfarin }\end{array}$ & & 0.31 & 0.40 & & 0.42 & & 0.30 & 0.47 & & 0.67 \\
\hline Major GI bleeding $(\% / y)$ & 1.02 & 1.12 & 1.51 & 0.86 & 0.76 & 1.23 & 0.82 & 1.51 & 2.16 & 3.15 \\
\hline HR for major GI bleeding & & $1.10^{*}$ & 1.50 & & $0.89 *$ & & 0.67 & 1.23 & & $P<.001 \ddagger$ \\
\hline
\end{tabular}

RE-LY, Randomized Evaluation of Long-Term Anticoagulation Therapy; ARISTOLE, Apixaban for Reduction in Stroke and Other Thromboembolic Events in Atrial Fibrillation; ENGAGE, ENGAGE AF-TIMI 48 (Effective Anticoagulation with Factor Xa Next Generation in Atrial Fibrillation-Thrombolysis in Myocardial Infarction 48); ROCKET AF, Rivaroxaban Once-daily Oral Direct Factor Xa Inhibition Compared with Vitamin K Antagonism for Prevention of Stroke and Embolism Trial in Atrial Fibrillation; bid, twice daily; HR, hazard ratio; GI, gastrointestinal. *HR calculation not significantly different. †Analysis according to $97.5 \%$ confidence interval. $\ddagger$ HR was not reported in the study’s report or assigned appendix.

This recommendation needs to be balanced with the return of bowel motility in cases of esophageal and abdominal surgery. In such cases, bridging anticoagulation with heparin or enoxaparin should be considered. A summary of the perioperative strategy is shown in Figure 1. For minor procedures with complete hemostasis, NOACs can be resumed 6 to 8 hours postoperatively. ${ }^{19}$

\section{COST OF NOACs}

As expected, the cost of NOACs is more expensive than that of warfarin, which has been on the market for decades. The annual cost of warfarin is $\$ 545$ (without INR testing), significantly less than the cost of dabigatran at $\$ 3650$, rivaroxaban at $\$ 3650$, and apixaban at $\$ 3445$.

However, the cost effectiveness has been extensively studied, especially for stroke prevention in patients with AF. The elimination of INR testing, decrease in adverse events, and decrease in the hospital length of stay will offset the increased drug costs of NOACs. Harrington and associates ${ }^{26}$ created a Markov analysis model using the data from large randomized controlled studies of NOACs. They compared the cost between warfarin and NOACs and calculated a willingness to pay threshold of $\$ 50,000$ per quality-adjusted life year gained for each medication. Although warfarin had the lowest cost $(\$ 77,813)$ followed by rivaroxaban $20 \mathrm{mg}$ $(\$ 78,738)$, dabigatran $150 \mathrm{mg}(\$ 82,719)$, and apixaban $5 \mathrm{mg}(\$ 85,326)$, the quality-adjusted life year gained was greatest for apixaban (8.47) followed by dabigatran (8.41), and rivaroxaban (8.26) and lowest for warfarin (7.97).

\section{FUTURE DIRECTIONS}

Cardiothoracic surgeons will increasingly encounter patients taking NOACs. The indications and use of NOACs are expected to increase as the knowledge and experience continues to build. Although the RE-ALIGN trial was stopped owing to the high complication rate, different regimens for NOACs in the setting of mechanical valves are anticipated. Other conditions that require anticoagulation, such as heparin-induced thrombocytopenia, could be treated in the future using NOACs. Future approval of a fourth NOAC, namely edoxaban, might eventually lead to a decrease in their cost and will be as cost-effective as warfarin. The development of reversal agents for NOACs will make management 
safer in urgent situations or in cases in which an overdose is suspected.

\section{CONCLUSIONS}

NOACs offer many advantages over warfarin. They are more convenient to use than warfarin with fewer drugdrug and food-drug interactions. Multiple, large, randomized clinical studies have confirmed the safety and efficacy of these NOACs. The clinical indications are increasing, such as venous thromboembolism prevention and treatment and the prevention of stroke in patients with nonvalvular AF. Currently, these drugs are not indicated for anticoagulation for a mechanical prosthesis. Cardiothoracic surgeons will face more patients taking these drugs in the future. Knowledge regarding the pharmacokinetic profile, interruption and restarting in the setting of surgery, and treatment in the case of severe bleeding is important for the treatment of patients taking this medication.

We would like to thank Drs Robert P. Giugliano and Christian T. Ruff for reviewing our report and giving us updated information.

\section{References}

1. Troconiz IF, Tillmann C, Liesenfeld KH, Schafer HG, Stangier J. Population pharmacokinetic analysis of the new oral thrombin inhibitor dabigatran etexilate (BIBR 148) in patients undergoing primary elective total hip replacement surgery. J Clin Pharmacol. 2007;47:371-82.

2. Paikin JS, Eikelboom JW, Cairns JA, Hirsh J. New antithrombotic agents—insights from clinical trials. Nat Rev Cardiol. 2010;7:498-509.

3. Hankey GJ, Eikelboom JW. Dabigatran etexilate: a new oral thrombin inhibitor. Circulation. 2011;123:1436-50.

4. Van de Werf F, Brueckmann M, Connolly SJ, Friedman J, Granger CB, Härtter S, et al. A comparison of dabigatran etexilate with warfarin in patients with mechanical heart valves: the randomized, phase II study to evaluate the safety and pharmacokinetics of oral dabigatran etexilate in patients after heart valve replacement (RE-ALIGN). Am Heart J. 2012;163:931-7.

5. Kubitza D, Becka M, Voith B, Zuehlsdorf M, Wensing G. Safety, pharmacodynamics, and pharmacokinetics of single doses of BAY 59-7939, an oral, direct factor Xa inhibitor. Clin Pharmacol Ther. 2005;78:412-21.

6. Eraso PLH, Merli GJ. Implications of new anticoagulants in primary practice. Int J Clin Pract. 2013;67:139-56.

7. Mendell J, Noveck RJ, Shi M. Pharmacokinetics of the direct factor Xa inhibitor edoxaban and digoxin administered alone and in combination. J Cardiovasc Pharmacol. 2012;60:335-41.

8. Flaker GC, Hohnloser S, Wojdyla D, Hylek E, Garcia D, Sullivan R, et al. Apicaban is efficacious and safe in patients with atrial fibrillation using concomitant amiodarone: an analysis from the ARISTOTLE trial. J Am Coll Cardiol. 2013;61(10_S).

9. Connolly SJ, Ezekowitz MD, Yusuf S, Eikelboom J, Oldgren J, Parekh A, et al. Dabigatran versus warfarin in patients with atrial fibrillation. $N$ Engl J Med. 2009:361:1139-51.
10. Connolly SJ, Ezekowitz MD, Yusuf S, Reilly PA, Wallentin L, Randomized Evaluation of Long-Term Anticoagulation Therapy Investigators Newly identified events in the RE-LY trial. N Engl J Med. 2010;363:1875-6.

11. Beasley BN, Unger EF, Temple R. Anticoagulant options-why the FDA approved a higher but not a lower dose of dabigatran. N Engl J Med. 2011; 364:1788-90.

12. Patel MR, Mahaffey KW, Garg J, Pan G, Singer DE, Hacke W, et al; ROCKET AF Investigators. Rivaroxaban versus warfarin in nonvalvular atrial fibrillation. N Engl J Med. 2011;365:883-91.

13. Patel MR, Mahaffey KW, Garg J, Pan G, Singer DE, Hacke W, et al. Apixaban in patients with atrial fibrillation. $N$ Engl J Med. 2011;364:806-17.

14. Giugliano RP, Ruff CT, Braunwald E, Murphy SA, Wiviott SD, Halperin JL, et al; ENGAGE AF-TIMI 48 Investigators. Edoxaban versus warfarin in patients with atrial fibrillation. N Engl J Med. 2013;369:2093-104.

15. Ruff CT, Giugliano RP, Braunwald E, Hoffman EB, Deenadayalu N, Ezekowitz MD, et al. Comparison of the efficacy and safety of new ora anticoagulants with warfarin in patients with atrial fibrillation: a meta-analysis of randomised trials. Lancet. 2014;383:955-62.

16. Eikelboom JW, Connolly SJ, Brueckmann M, Granger CB, Kappetein AP, Mack MJ, et al; the RE-ALIGN Investigators. Dabigatran versus warfarin in patients with mechanical heart valves. $N$ Engl J Med. 2013;369: 1206-14.

17. January CT, Wann LS, Alpert JS, Calkins H, Cleveland JC Jr, Cigarroa JE et al. 2014 AHA/ACC/HRS guideline for the management of patients with atrial fibrillation: executive summary: a report of the American College of Cardiology/American Heart Association Task Force on Practice Guidelines and the Heart Rhythm Society. Circulation. April 10, 2014 [Epub ahead of print].

18. Camm AJ, Lip GY, De Caterina R, Savelieva I, Atar D, Hohnloser SH, et al; ESC Committee for Practice Guidelines (CPG). 2012 Focused update of the ESC guidelines for the management of atrial fibrillation: an update of the 2010 ESC guidelines for the management of atrial fibrillation: developed with the special contribution of the European Heart Rhythm Association. Eur Heart J. 2012;33:2719-47.

19. Bergmark B, Giugliano RP. Perioperative management of target-specific oral anticoagulants. Hosp Pract (1995). 2014;42:38-45.

20. Spyropoulos AC, Douketis JD. How I treat anticoagulated patients undergoing an elective procedure or surgery. Blood. 2012;120:2954-62.

21. Lai A, Davidson N, Galloway SW, Thachil J. Perioperative management of patients on new oral anticoagulants. Br J Surg. 2014;101:742-9.

22. Dager WE, Gosselin RC, Kitchen S, Dwyre D. Dabigatran effects on the international normalized ratio, activated partial thromboplastin time, thrombin time, and fibrinogen: a multicenter, in vitro study. Ann Pharmacother. 2012; 46:1627-36

23. Samama MM, Martinoli JL, LeFlem L, Guinet C, Plu-Bureau G, Depasse F, et al Assessment of laboratory assays to measure rivaroxaban — an oral, direct factor Xa inhibitor. Thromb Haemost. 2010;103:815-25.

24. Marlu R, Hodaj E, Paris A, Albaladejo P, Crackowski JL, Pernod G. Effect of nonspecific reversal agents on anticoagulant activity of dabigatran, rivaroxaban: a randomised crossover ex vivo study in healthy volunteers. Thromb Haemost 2012;108:217-24.

25. Hughes S. Available at: http://www.medscape.com/viewarticle/818169. Accessed December 20, 2013.

26. Harrington AR, Armstrong EP, Nolan PE Jr, Malone DC. Cost-effectiveness of apixaban, dabigatran, rivaroxaban, and warfarin for stroke prevention in atrial fibrillation. Stroke. 2013;44:1676-81.

27. Douketis JD, Spyropoulos AC, Spencer FA, Mayr M, Jaffer AK, Eckman MH, et al. Therapy and prevention of thrombosis, 9th ed: American College of Ches Physicians evidence-based clinical practice guidelines. Chest. 2012; 141(2 Suppl):e326S-50S. 


\section{APPENDIX 1 \\ Definition of Pharmacologic Terms}

Bioavailability - the rate and extent to which the active ingredient or active moiety is absorbed (gastrointestinal) from a drug product and becomes available at the site of action (Food and Drug Administration [FDA] definition)

Prodrug - a biologically inactive compound that does not produce pharmacologic effects until it is metabolized within the body

T1/2-half-life (the time required for the amount or concentration of a drug in the body to be reduced to exactly one-half of a given amount or concentration)

p-gp protein-permeability glycoprotein (cell membrane transporter, operating as an adenosine triphosphate-powered drug efflux pump, present in high concentrations on the apical surface of the epithelial cell lining of the small intestine and colon and in other epithelial and endothelial cell linings

p-gp protein inhibitors-amiodarone, azithromycin, captopril, clarithromycin, cyclosporine, ketoconazole, quinidine, quinine, ritonavir, verapamil, and tacrolimus

CYP3A4-a member of the cytochrome P450 mixed-function oxidase system, CY3A4 is one of the most important enzymes involved in the metabolism of xenobiotics (small foreign organic molecules) in the human body

CYP3A in vivo inducers (FDA)

Strong inducers: carbamazepine, phenytoin, rifampin, St John's wort

Moderate inducers: bosentan, efavirenz, etravirine, modafinil, nafcillin

Weak inducers: amprenavir, aprepitant, armodafinil, pioglitazone, prednisone, rufinamide

CYP3A4 inhibitors (FDA)

Rifampin and carbamazepine

CYP3A in vivo inhibitors (FDA)

Strong inhibitors: clarithromycin, conivaptan, grapefruit juice (depending on the concentrate), indinavir, itraconazole, ketoconazole, lopinavir/ritonavir, nefazodone, nelfinavir, posaconazole, telithromycin, voriconazole

Moderate inhibitors: ciprofloxacin, diltiazem, erythromycin, fluconazole, fosamprenavir, grapefruit juice (depending on the concentrate), imatinib, verapamil, amprenavir, aprepitant, darunavir/ritonavir

Weak inhibitors: alprazolam, amiodarone, amlodipine, atorvastatin, bicalutamide, cilostazol, cimetidine, cyclosporine, fluoxetine, fluvoxamine, isoniazid, nilotinib, oral contraceptives, ranitidine, ranolazine, ripranavir/ritonavir, zileuton

CYP3A4-specific inhibitors (FDA)

Atazanavir, clarithromycin, indinavir, itraconazole, ketoconazole, nefazodone, nelfinavir, ritonavir, saquinavir, telithromycin

CYP3A and p-gp dual inhibitors (FDA)

Cyclosporine A, ketoconazole, nelfinavir, ritonavir, saquinavir

Strong CYP3A inhibitor and p-gp inhibitors (FDA): itraconazole, lopinavir/ritonavir, clarithromycin, ritonavir, ketoconazole, indinavir/ ritonavir, conivaptan

Medium CYP3A inhibitor and p-gp inhibitors (FDA): verapamil, erythromycin, diltiazem, dronedarone

Weak CYP3A inhibitor and p-gp inhibitors: quinidine, ranolazine, amiodarone, felodipine, azithromycin

Narrow therapeutic index drugs: drugs that have less than a twofold difference in the minimum toxic concentration and minimum effective concentration in the blood

Wide therapeutic index drugs: drugs that have a greater than fivefold difference between the minimum toxic concentration and the minimum effective concentration in the blood

Quality-adjusted life year: measure of health improvement used by healthcare providers to guide healthcare resource allocation decisions; better described as years weighted by their quality; consequently, it takes into account both the quality and the quantity of life generated by an intervention

\section{Warfarin Interactions}

Drugs

Increasing warfarin's effect: acetaminophen, aspirin, allopurinol, amiodarone, cephalosporin, ciprofloxacin, clopidogrel, diclofenac, fluconazole, metronidazole, omeprazol, tamoxifen

Decreasing warfarin's effect: azathioprine, antithyroid drugs, carbamazepine, haloperidol, oral contraceptives, phenobarbital, rifampin, vitamin $\mathrm{K}$

Food: cranberry, vitamin K-containing foods (eg, broccoli, spinach, kale, lettuce, Brussels sprouts, cabbage) 